\title{
Differential Effects of Physical and Social Enriched Environment on Angiogenesis in Male Rats After Cerebral Ischemia/Reperfusion Injury
}

\section{OPEN ACCESS}

Edited by:

Anna-Sophia Wahl,

University of Zurich, Switzerland

Reviewed by:

Hüseyin Akan,

Ondokuz Mayıs University, Turkey Julia Kaiser,

Burke Neurological Institute (BNI), United States

*Correspondence: Wei-Jing Liao weijingliaorehab@163.com Xiu-Ping Chen xiupingchenrehab@163.com

Specialty section:

This article was submitted to Health,

a section of the journa Frontiers in Human Neuroscience

Received: 29 October 2020 Accepted: 02 March 2021

Published: 26 March 2021

Citation:

Zhang X, Liu J-Y, Liao W-J and Chen X-P (2021) Differential Effects

of Physical and Social Enriched Environment on Angiogenesis in Male Rats After Cerebral

Ischemia/Reperfusion Injury. Front. Hum. Neurosci. 15:622911. doi: 10.3389/fnhum.2021.622911

\author{
Xin Zhang ${ }^{1}$, Jing-Ying Liu' ${ }^{1}$, Wei-Jing Liao ${ }^{*}$ and Xiu-Ping Chen ${ }^{2 *}$ \\ ${ }^{1}$ Department of Rehabilitation Medicine, Zhongnan Hospital of Wuhan University, Wuhan, China, ${ }^{2}$ Department \\ of Rehabilitation Medicine, The first Affiliated Hospital of Nanchang University, Nanchang, China
}

Different housing conditions, including housing space and the physiological and social environment, may affect rodent behavior. Here, we examined the effects of different housing conditions on post-stroke angiogenesis and functional recovery to clarify the ambiguity about environmental enrichment and its components. Male rats in the model groups underwent right middle cerebral artery occlusion (MCAO) followed by reperfusion. The MCAO rats were divided into four groups: the physical enrichment (PE) group, the social enrichment (SE) group, the combined physical and social enrichment (PSE) group and the ischemia/reperfusion + standard conditioning (IS) group. The rats in the sham surgery (SS) group were housed under standard conditions. In a set of behavioral tests, including the modified Neurological Severity Score (mNSS), rotarod test, and adhesive removal test, we demonstrated that the animals in the enriched condition groups exhibited significantly improved neurological functions compared to those in the standard housing group. Smaller infarction volumes were observed in the animals of the PSE group by MRI detection. The enriched conditions increased the microvessel density (MVD) in the ischemic boundary zone, as revealed by CD31 immunofluorescent staining. The immunochemical and q-PCR results further showed that environmental enrichment increased the expression levels of angiogenic factors after ischemia/reperfusion injury. Our data suggest that all three enrichment conditions promoted enhanced angiogenesis and functional recovery after ischemia/reperfusion injury compared to the standard housing, while only exposure to the combination of both physical and social enrichment yielded optimal benefits.

Keywords: ischemia/reperfusion, enriched environment, angiogenesis, VEGF, ANG-1, Tie-2

\section{INTRODUCTION}

An enriched environment (EE) classically consists of a large amount of space, social enrichment (SE), and diverse multisensory stimulation, which are supposed to facilitate enhanced sensory, cognitive, motor, and social stimulation compared to those provided by standard laboratory housing conditions (Hannan, 2014). EE provides animals with optimal conditions for enhanced exploration, cognitive activity, social interaction, and physical exercise (Wadowska et al., 2015). Early studies have demonstrated that EE treatment elicits many positive effects at the molecular, anatomical, and functional levels after brain injury (Cassarino and Setti, 2015). However, despite 
the largely positive evidence, non-significant or even negative effects of EE have also been reported (Fuss et al., 2010; Walker and Mason, 2011). The reason for these inconsistent results could be due to the variety in EE settings. Given that EE is a comparative concept and includes several key components (social interaction, cognitive activity and physical exercise), one important question that arises from EE studies is the extent to which the different components of EE can be separated and analyzed with respect to their beneficial effects. The most studied aspect has been physical exercise. In animal experiments, there are two kinds of exercise interventions: voluntary and forced (Luo et al., 2007; Zhang et al., 2010). Many studies have shown that increased voluntary physical exercise can enhance cognition and alter motor states in wild-type rodents and may induce some cellular changes after stroke (Pang and Hannan, 2013; Voss et al., 2013). Grégoire et al. (2014) confirmed the central importance of exercise in EE when they evaluated the impacts of individual variables of EE on adult hippocampal neurogenesis in healthy adult male CD1 mice. However, Johansson and Ohlsson (1996) reported that the effects of physical enrichment (PE) (exercise) in the form of wheel running on motor performance were inferior to those of social interaction. They also confirmed that an EE allowing free physical activity combined with social interaction resulted in the best performance in ischemic rats. The PE condition in this study added a number of complex auditory, olfactory, visual, and tactile stimuli for the animals, which were intended to induce voluntary exercise. The SE condition provided the animals with more social interaction and relatively more sensorimotor stimulation by housing more animals together in a larger space. Social enrichment has also been proven to have a beneficial effect on neurogenesis (Venna et al., 2014), while social isolation has adverse effects on cognition (Fone and Porkess, 2008). Previous animal studies also indicated that social stimulation is an independent factor that promotes beneficial effects on the central nervous system by reducing anxiety-like behaviors, stimulating voluntary exercise, promoting neurogenesis and facilitating functional recovery following stroke (Fowler et al., 2002; Silasi et al., 2008; Cirulli et al., 2010; Xie et al., 2013). From a theoretical perspective, Will and associates (Will et al., 2004) argued that the social component of the EE paradigm increased exploration and locomotor activity, as the rats "play" in an interactive milieu. Diverse EE paradigms are used between laboratories, with little understanding of how individual EE variables (such as social interactions and physical exercise) might differentially impact specific downstream biological mechanisms.

Angiogenesis is a form of neurovascular remodeling of great importance in numerous pathological conditions, including stroke. Post-stroke angiogenesis not only increases collateral circulation and restores oxygen and nutrient supply to the injured tissue but also provides neurotrophic support to concurrent neurogenesis and synaptogenesis, which all ultimately lead to long-term functional recovery (Petcu et al., 2010). Substantial evidence has shown that neurogenesis and angiogenesis after ischemic stroke are coupled processes and should be acknowledged and pursued as concurrent and nonmutually exclusive events to develop further neurorestorative therapy strategies (Font et al., 2010; Lacar et al., 2012; Ruan et al., 2015). A previous study showed that voluntary physical activity improves long-term stroke outcome in wild-type mice and that this effect was related to augmentation of angiogenesis and cerebral blood flow within the ischemic striatum (Gertz et al., 2006). Our previous research as well as other studies have demonstrated that typical EE enhanced angiogenesis and reduced neurologic deficits in ischemic rats (Seo et al., 2013; Yu et al., 2014; Zhang et al., 2017). However, the housing paradigms in these studies could not unambiguously evaluate the effects of separate EE variables, such as social interaction and physical exercise, which were also reported to influence angiogenesis and neurogenesis (Leasure and Decker, 2009; Zhang et al., 2013). Clearly defining the relative and/or combinatorial effects of such variables is essential for the design of EE paradigms for both research and rehabilitative use.

In this study, we established a set of different housing conditions to experimentally isolate the social interaction and physical exercise provided by typical EE with the aim of gaining insights into their isolated and combined contributions to functional recovery and angiogenesis after stroke.

\section{MATERIALS AND METHODS}

\section{Animals}

A total of 78 healthy adult male Sprague-Dawley rats (purchased from the Experimental Animal Center of Wuhan University, Wuhan, Hubei, China) weighing 220-240 g were housed in a controlled environment $\left(20 \pm 1^{\circ} \mathrm{C}, 55 \pm 5 \%\right.$ relative humidity, light period from 8:00 to 20:00) with free access to fresh water and standard rat chow. In order to exclude the effect of gender on stroke recovery, only male rats were selected for the study (Koellhoffer and McCullough, 2013). After arrival, all rats underwent 3 days of beam-walking, rotarod, and adhesive removal training to prepare for future neurological function tests. Following training, the animals were numbered and randomized into different groups by using a table of random numbers. Then the rats were randomly assigned to one of the following groups: the ischemia/reperfusion (I/R) + SE group $(n=16)$, the ischemia/reperfusion + PE group $(n=16)$, the ischemia/reperfusion + physical and social enrichment group (PSE, $n=16$ ), the ischemia/reperfusion + standard condition group (IS, $n=16$ ) and the sham + standard condition group (SS, $n=14$ ). The experimental protocol and group settings are shown in Figure 1A. All animal experimental procedures were approved according to the animal experimental committee of Wuhan University at Wuhan, China. Every effort was made to minimize the number of animals used and their suffering. Animals were anesthetized with inspired concentrations of $4 \%$ (induced) and $2 \%$ (maintained) isoflurane. The brains were transcranial perfused with $200 \mathrm{~mL}$ of $0.9 \%$ saline followed by perfusion and immersion in $4 \%$ paraformaldehyde solution. The excluded animals were euthanized with inhalation of CO2. There were no surviving animals at the end of study. 

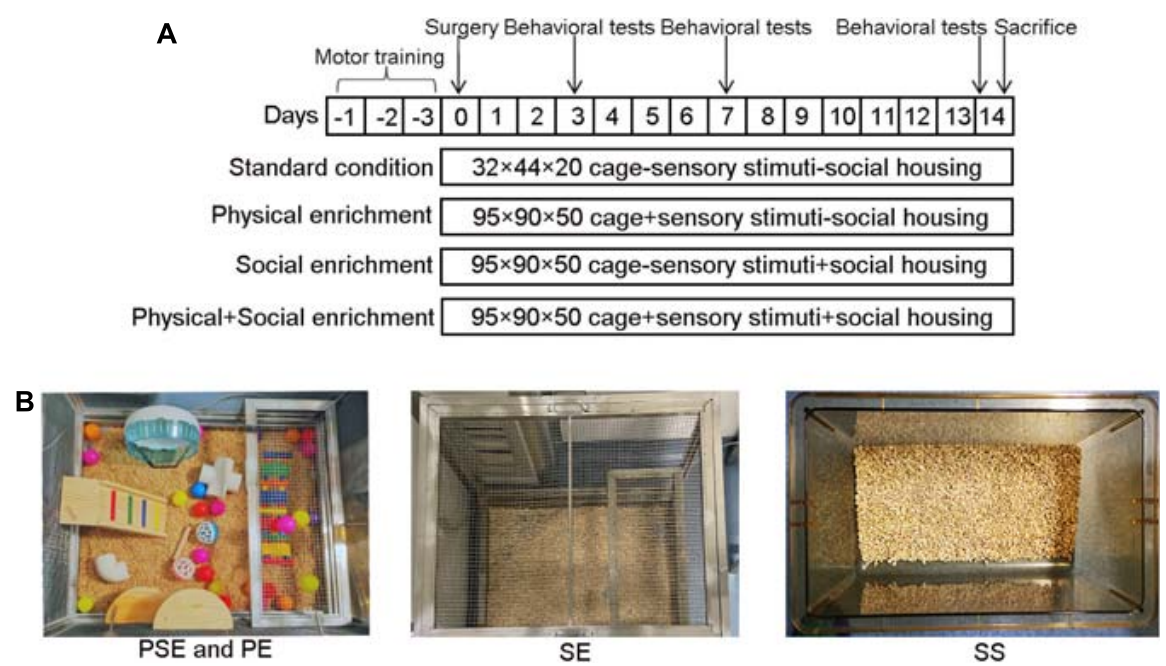

FIGURE 1 | Flow chart of the experimental protocol and enrichment settings. (A) The experimental protocol and group settings. (B) The setting of PSE, PE, SE, and SS.

\section{Surgery}

Occlusion of the right middle cerebral artery was performed using the modified intraluminal filament technique. All procedures were performed according to the Guidelines of the National Institutes of Health Guide for the Care and Use of Laboratory Animals. Surgical anesthesia was induced and maintained with inspired isoflurane at concentrations of 4 and $2 \%$, respectively, in $2: 1 \mathrm{~N}_{2} \mathrm{O}: \mathrm{O}_{2}$. The body temperature was maintained at $37.0 \pm 0.5^{\circ} \mathrm{C}$ with warming pads. After a $2 \mathrm{~cm}$ median incision of the neck skin, the right carotid artery (CCA), external carotid artery (ECA), and internal carotid artery (ICA) were carefully isolated. After that, a monofilament nylon filament (Beijing Cinontech Biotech Co., Ltd., Beijing, China) was inserted through the right CCA and gently advanced into the ICA to a point approximately $18 \mathrm{~mm}$ distal to the bifurcation of the carotid artery, and the right MCA was then occluded. After $2 \mathrm{~h}$, the filament was gently withdrawn for reperfusion. In the sham surgery (SS) group, all surgical procedures were the same except a nylon filament was not inserted (Longa et al., 1989). While the time of SS was 3 min shorter on average than that of the surgery group, no difference was found in the total anesthesia duration between sham animals and I/R animals. Three rats did not survive the surgery due to excessive bleeding because the vagus nerve was accidentally cut while separating it from the ICA.

\section{Acute Neurological Evaluation}

After the rats recovered from anesthesia, the neurologic deficit findings were scored on a five-point scale to determine whether the middle cerebral artery occlusion (MCAO) model was successfully induced (Longa et al., 1989). Rats with scores of 13 were considered successful models and were included in the study. Six rats with scores of 0 , which indicated no neurologic deficit, and four rats with scores of 4, which indicated a depressed level of consciousness, were excluded. Five rats were excluded during the 2-week follow-up due to cervical hematoma compression or subarachnoid hemorrhage; thus, the ultimate number of rats in each group was 12 . All of the excluded animals were euthanized by $\mathrm{CO} 2$ inhalation to minimize their suffering, and the procedures were approved according to the Institutional Animal Care and Use Committee of Wuhan University.

\section{Housing Conditions}

One day after MCAO, rats of different groups were returned to their corresponding cages. The rats in the PE, SE, and PSE groups were kept in the corresponding environments for $4 \mathrm{~h}$ in the morning and were kept under standard conditions for the rest of the experiment. The rats in the SE groups were always grouped with the same rats in the social EE. The rats in the IS and SS groups received continuous standard housing. Additionally, the IS and SS rats were moved for $4 \mathrm{~h}$ from their usual standard cage to a different standard cage to equalize the handling procedures of all rats. The details of the different conditions were as follows:

\section{Standard Conditions}

The rats were housed in standard laboratory cages that were $32 \mathrm{~cm}$ wide $\times 44 \mathrm{~cm}$ long $\times 20 \mathrm{~cm}$ high with nothing but bedding materials inside. The rats were housed in groups of two.

\section{Socially Enriched Environment}

Animals were housed in a $75 \mathrm{~cm}$ wide $\times 90 \mathrm{~cm}$ long $\times 50 \mathrm{~cm}$ high stainless-steel network surface cage with bedding materials inside. Six to ten rats were housed together in one cage. And they were always grouped with the same rats in the social EE.

\section{Physically Enriched Environment}

Animals were housed in a $75 \mathrm{~cm}$ wide $\times 90 \mathrm{~cm}$ long $\times 50 \mathrm{~cm}$ high stainless-steel network surface cage with bedding materials, several differently-colored plastic balls, differently-shaped wooden blocks, a T-shaped and an L-shaped plastic tunnel, climbing ladders and a running wheel inside for exploration and 
exercise. Three times a week, the plastic balls and wooden blocks were changed with new items of different colors or shapes, and the tunnels, ladders, and running wheel were relocated. The rats were housed in groups of two.

\section{Physically and Socially Enriched Environment}

The cage and objects were the same as the physically EE, while the rats were housed in groups of 6-10. The water and food supply were the same for all animals (Figure 1B).

\section{Neurological Function Tests}

A set of behavior tests, including the Modified Neurological Severity Score (mNSS), rotarod test and adhesive removal test, were carried out at 3, 7, and 14 days after MCAO by an investigator who was unaware of the experimental groups.

The mNSS was scored on a scale of 0-18 (no deficit score, 0 ; maximal deficit score, 18) and included a composite of tests to evaluate motor (muscle status, abnormal movement), sensory (visual, tactile, and proprioceptive), reflex, and balance abilities. One point was scored for the failure to perform a certain task or the absence of a particular reflex. Thus, a higher score indicated a more severe injury (Jin et al., 2014).

A rotarod test with accelerating velocity was employed to measure balance and coordination deficits of rats (LE8200 Panlab, Harvard Apparatus, United States). During the test, accelerating velocity (4-40 rpm) was achieved within $260 \mathrm{~s}$ with a maximal testing time of $300 \mathrm{~s}$. The time until the animals dropped was measured. Tests were always performed twice, and the means were used for statistical analysis (Doeppner et al., 2014).

The adhesive removal test was performed to measure somatosensory deficits. Briefly, two small pieces of adhesivebacked paper dots (of equal size, $113.1 \mathrm{~mm}^{2}$ ) were used as bilateral tactile stimuli occupying the distal-radial region on the wrist of each forelimb. The time required to remove the stimulus from each limb was recorded. Each animal received five trials at 3, 7, and 14 days after surgery, and the mean time required to remove both stimuli from the limbs was recorded (Diederich et al., 2014).

\section{Magnetic Resonance Imaging for Infarct Volume}

We used a Bruker 7.0T magnetic resonance imaging (MRI) horizontal scanner (Institute of Physics and Mathematics, Chinese Academy of Sciences, Wuhan, China) to quantify the lesion volume at 14 days after MCAO ( $n=6 /$ group). The rats were anesthetized with inspired concentrations of $4 \%$ (induced) and $2 \%$ (maintained) isoflurane. Coronal images were obtained with the central slice $0.5 \mathrm{~mm}$ from bregma. T2-weighted multislice images were acquired using a RARE sequence with the following parameters: time-to-repetition $(\mathrm{TR})=3,000 \mathrm{~ms}$, effective timeto-echo $=36 \mathrm{~ms}$, RARE factor $=8$, matrix size $=256 \times 256$, field-of-view $=30 \mathrm{~mm} \times 30 \mathrm{~mm}$, and $\mathrm{BR}=24$ slices with a slice thickness of $0.8 \mathrm{~mm}$. For further processing, MRI sequences were exported as DICOM sequences. The volume of ischemic lesions was determined by a blinded investigator using ImageJ software (NIH, Bethesda, MD, United States). The total infarct area was obtained by adding the infarct areas of all sections and multiplying the value by the slice thickness to derive the infarct volume. The percentage of infarct volume was calculated by the following equation: percentage of infarct volume $=$ (infarct volume/contralateral hemispheric volume) $\times 100 \%$.

\section{Immunofluorescent Staining}

Brain paraffin sections $(4 \mu \mathrm{m})$ were performed heat mediated antigen retrieval with Tris/EDTA buffer for $20 \mathrm{~min}$ after hydrated. The sections were treated with $0.1 \%$ Triton-X for 10 min and blocked with $10 \%$ FBS for $1 \mathrm{~h}$ and then primarily incubated with mouse monoclonal to CD31 antibody (1:100, Abcam, Cambridge, United Kingdom; product code: ab119339, RRID: AB_10936456; Immunogen: Human CD31) at $4^{\circ} \mathrm{C}$ overnight. After washing, the brain sections were then incubated with Cy3-conjugated goat anti-mouse IgG secondary antibody (1:50, Jackson ImmunoResearch Labs, West Grove, PA, United States; Product Code: 115-166-003, RRID: AB_2338699) for $1 \mathrm{~h}$. For quantitative measurements of microvessel density (MVD), five slides from each brain, with each slide containing four fields $(\times 200)$ from the penumbra, were captured under a fluorescence microscope (Olympus Corporation, Tokyo, Japan) and analyzed using ImageJ software (NIH, Bethesda, MD, United States). The interval between the sections was $200 \mu \mathrm{m}$. Ischemic boundary zone was defined as a region of $300 \mu \mathrm{m}$ distal to the stroke core with hypovascularization (Figure 4C; Rust et al., 2019). Any endothelial cell or group of cells that stained positive for CD31 and was distinct from neighboring tissues was counted as a microvessel. The average of the values obtained by the two reviewers for each field was reported as a single numerical value, and the mean count from the five regions was used to determine the MVD score.

\section{Immunohistochemical Assessment}

Rats ( $n=6$ /group) were killed at 14 days after MCAO. The brains were transcardially perfused with $200 \mathrm{~mL}$ of $0.9 \%$ saline followed by perfusion and immersion in $4 \%$ paraformaldehyde solution before being embedded in paraffin. A series of $4 \mu \mathrm{m}$ thick brain sections were sliced from the paraffin block for immunohistochemistry to detect the expression of angiopoietin1 (Ang1) and its receptor Tie-2. The procedure was performed as reported previously (Cui et al., 2009). Brain sections were hydrated and performed heat mediated antigen retrieval with Tris/EDTA buffer for $20 \mathrm{~min}$ before commencing with IHC staining protocol. The slices were then treated with $3 \%$ $\mathrm{H}_{2} \mathrm{O}_{2}$ for 10 min and blocked with $1 \%$ BSA for $1 \mathrm{~h}$. And the samples were incubated overnight at $4^{\circ} \mathrm{C}$ with a rabbit polyclonal antibody against angiopoietin 1 (1:100, Abcam, Cambridge, United Kingdom; Product Code: ab-102015, RRID: AB_10712377; Immunogen: Synthetic peptide corresponding to human angiopoietin 1 internal sequence aa 225-239), rabbit polyclonal antibody against Tie-2 (1:100, Santa Cruz Biotechnology, Inc., Dallas, TX, United States; Product Code: sc9026, RRID: AB_2203226; Immunogen: Extracellular domain of human Tie-2 aa 25-200). After three rinses (5 min each) with phosphate-buffered saline ( $\mathrm{PBS}, \mathrm{pH}=7.4$ ), the sections were reacted with peroxidase-labeled anti-rabbit IgG (1:200, Dako, Agilent Pathology Solutions, Santa Clara, CA, United States; 
Product Code: K4003, RRID: AB_2630375). Negative controls were established by replacing the primary antibody with PBS. For qualitative analysis, four visual fields $(\times 200)$ from each section in the penumbra were captured under a light microscope (Olympus Corporation, Tokyo, Japan).

\section{Real-Time Quantitative (q)-and Reverse Transcription (RT)-PCR}

Rats ( $n=6$ /group) were decapitated after MRI examination, and cortical tissue samples were collected. qRT-PCR was performed to evaluate vascular endothelial growth factor (VEGF) gene expression. Total RNA was extracted from the cerebral cortex of the penumbra using TRIzol (Invitrogen, United States). Adding trichloromethane into the homogenate. The mixture was centrifuged at $10,000 \mathrm{~g}$ at $4^{\circ} \mathrm{C}$ for $10 \mathrm{~min}$. Isopropanol precooled at an equal volume of $4^{\circ} \mathrm{C}$ was added, mixed upside down, and stood at $-20^{\circ} \mathrm{C}$ for $15 \mathrm{~min}$. Then the solution was centrifuged at $10,000 \mathrm{~g}$ at $4^{\circ} \mathrm{C}$ for $10 \mathrm{~min}$. Pre-cooled $75 \%$ ethanol was added at $4^{\circ} \mathrm{C}$ and reversed for several times. The RNA was washed and centrifuged at $10,000 \mathrm{~g}$ at $5 \mathrm{~min}$. After drying for several minutes, ethanol was fully volatilized and RNA was fully dissolved in Rnase-free Water. Total RNA was reverse transcribed using a First Strand cDNA Synthesis Kit (Toyobo, Japan). Realtime PCR was performed using SYBR ${ }^{\circledR}$ Premix Ex Taq ${ }^{\text {TM }}$ Mix (TaKaRa, Japan) in a StepOne ${ }^{\mathrm{TM}}$ Real-Time PCR System (Life Technologies). The following PCR conditions were used: $95^{\circ} \mathrm{C}$ for $1 \mathrm{~min}$, followed by $40 \mathrm{cycles}$ of $95^{\circ} \mathrm{C}$ for $15 \mathrm{~s}, 58^{\circ} \mathrm{C}$ for $20 \mathrm{~s}$, and $72^{\circ} \mathrm{C}$ for $45 \mathrm{~s}$. The forward and reverse primer sequences used for real-time PCR were as follows:

\section{VEGF (F): 5' - TGTGAGCCTTGTTCAGAGCG -3'; VEGF (R): 5' - GACGGTGACGATGGTGGTGT -3'; Ang-1(F): $5^{\prime}$-TAACCTCGCCCTGCAAAGAG-3'; Ang-1(R): $5^{\prime}$-CTGTATGCTTGCAGGTGGTGAT-3'; GAPDH (F): $5^{\prime}$ - CGCTAACATCAAATGGGGTG - $3^{\prime}$; GAPDH (R): 5' - TTGCTGACAATCTTGAGGGAG - $3^{\prime}$.}

\section{Statistical Analysis}

Statistical analyses were performed using SPSS 20.0 statistical software. The behavioral test scores were analyzed with conventional two-factor analyses of variance (ANOVA) followed by Bonferroni's post hoc tests. The other data were analyzed using one-way ANOVA followed by Bonferroni's post hoc tests. All data were normally distributed and are presented as the mean \pm standard deviation (SD). Normal distribution was evaluated by the Kolmogorov-Smirnov test. A value of $P<0.05$ was considered significant.

\section{RESULTS}

\section{Enriched Conditioning Improved Neurological Functional Outcomes}

We first compared the effects of different housing conditions on motor deficits using the mNSS, rotarod test and adhesive removal test at 3, 7, and 14 days after MCAO. Twoway ANOVA revealed significant differences due to time
$\left[F_{(2,88)}=287.65, n=60, P<0.001\right]$, group $\left[F_{(4,88)}=475.96\right.$, $n=60, P<0.001]$, and the interaction between these two factors $\left[F_{(8,88)}=26.19, n=60, P<0.001\right]$ in the mNSS results. Rats in the sham + standard condition group had mild neurological symptoms but fully recovered 3 days after the SS. There were no significant differences in mNSS results among the ischemia/reperfusion groups (the ischemia/reperfusion $+\mathrm{PE}$ group, the ischemia/reperfusion $+\mathrm{SE}$ group, the ischemia/reperfusion + PSE group and the ischemia/reperfusion + standard condition group) on day 3 $(P>0.05)$. The ischemia/reperfusion rats exhibited progressive functional recovery on day 7 , but the ischemia/reperfusion $+\mathrm{PE}$ group, the ischemia/reperfusion $+\mathrm{SE}$ group and the ischemia/reperfusion + PSE group exhibited better recovery than the ischemia/reperfusion + standard condition group $(P<0.001$ vs. $\mathrm{PE}, P=0.001$ vs. $\mathrm{SE}, P<0.001$ vs. PSE). On day 14 , the mNSS scores of the three enriched conditioning groups were significantly lower than those of the ischemia/reperfusion + standard condition group $(P<0.001)$. The rats in the ischemia/reperfusion $+\mathrm{PE}$ group showed a much better functional recovery than those in the ischemia/reperfusion $+\mathrm{SE}$ group $(P=0.003)$, while the ischemia/reperfusion + PSE group rats achieved the best recovery among the three enriched groups $(P=0.007$ vs. $P E, P<0.001$ vs. SE, Figure 2A).

For the rotarod test, there were significant differences due to group $\left[F_{(4,88)}=901.65, n=60, P<0.001\right]$, time $\left[F_{(2,88)}=760.55, n=60, P<0.001\right]$, and the group $\times$ time interaction $\left[F_{(8,88)}=30.10, n=60, P<0.001\right]$. Rats under enriched conditions exhibited significantly increased time on the rod at 7 and 14 days after MCAO compared to those under standard conditions $(P<0.001)$, while rats in both the ischemia/reperfusion + PSE group and the ischemia/reperfusion $+\mathrm{PE}$ group showed a much better functional status than those in the ischemia/reperfusion $+\mathrm{SE}$ group at day 14 ( $P<0.001$ vs. $P S E, P=0.006$ vs. $P E$, Figure 2B).

For the adhesive removal test, significant differences were seen due to time $\left[F_{(4,88)}=261.93, n=60, P<0.001\right]$, group $\left[F_{(4,88)}=174.69, n=60, P<0.001\right]$, and the interaction between these two factors $\left[F_{(4,88)}=15.15, n=60, P<0.001\right]$. At day 7 after MCAO, only the PSE condition led to significantly decreased time required to remove the stimuli compared to the standard condition $(P=0.008)$. At day 14 , the rats in the three enriched groups exhibited better functional results than the rats in the ischemia/reperfusion + standard condition group $(P<0.001)$, while the effect of the PSE condition outweighed those of the $\mathrm{PE}$ and the SE conditions $(P=0.007$ vs. $P E, P=0.013$ vs. $S E$, Figure 2C).

\section{Physical Enrichment and the Combination of Physical and Social Enrichment Reduced Infarct Volume in Ischemia/Reperfusion Rats}

To elucidate whether enhanced neurological recovery was a consequence of structural neuroprotection, we analyzed infarct volumes 14 days after stroke. As demonstrated by MRI 


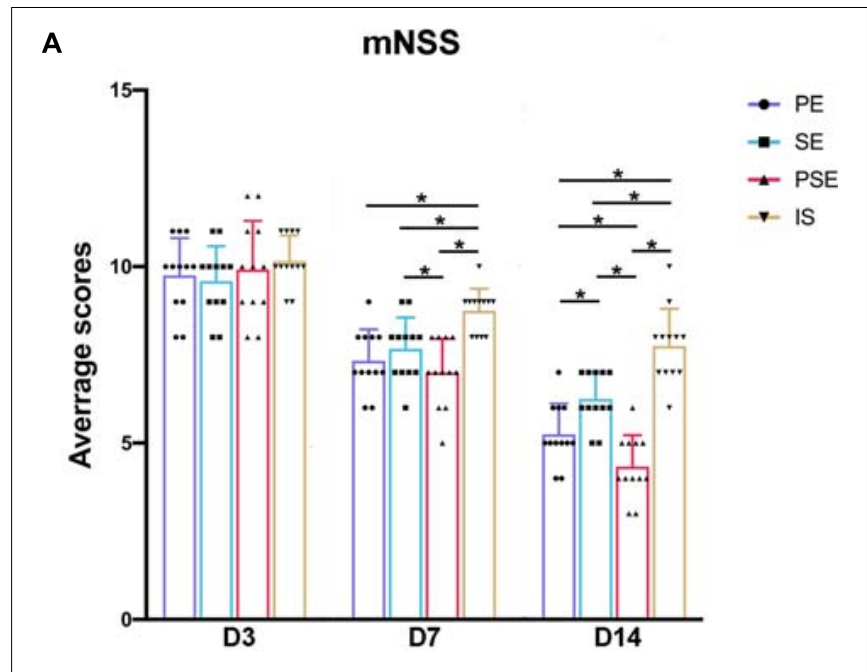

B

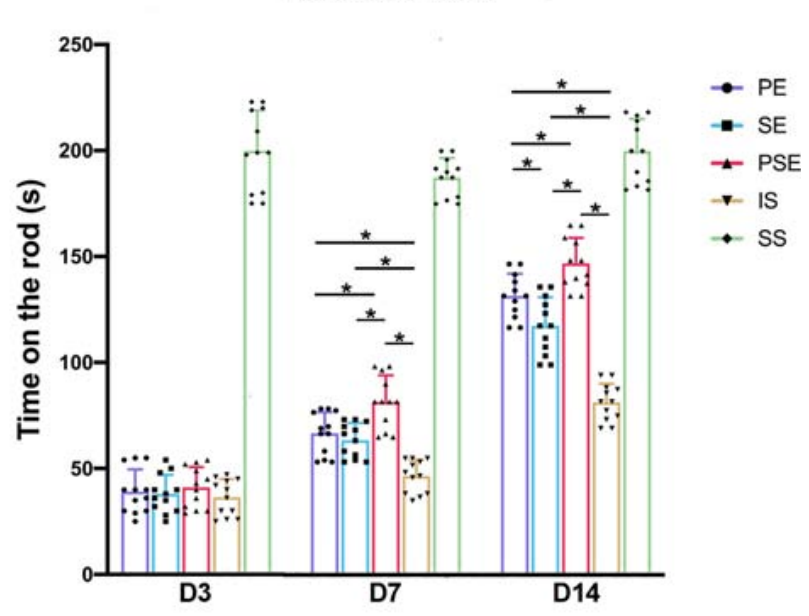

C

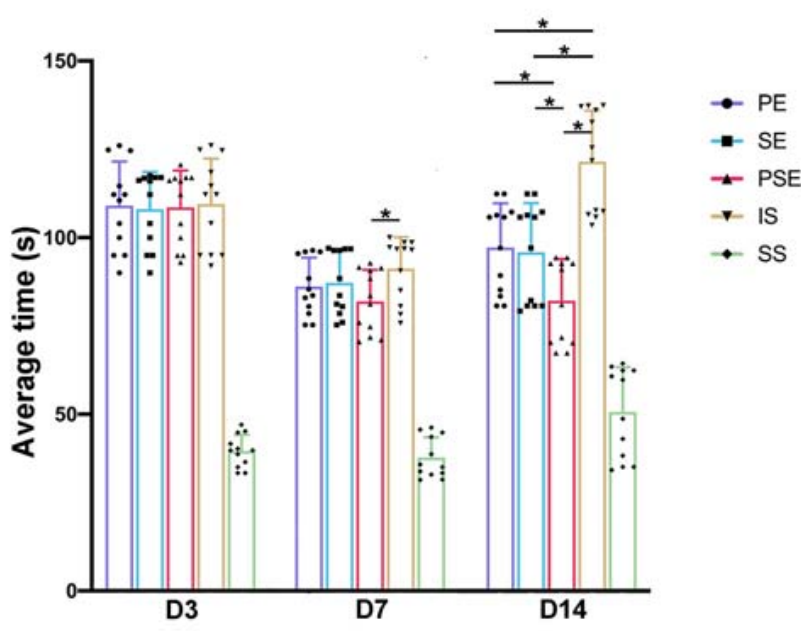

FIGURE 2 | Behavioral tests. The results of the modified Neurological Severity Score (mNSS) (A) rota-rod test (B) and adhesive removal test (C) on 3, 7, and 14 days of PE, SE, PSE, IS, and SS group. $n=12$. Data are expressed as mean \pm SEM; ${ }^{*} P<0.05$. images (Figure 3A), there was a significant difference among different housing conditions in infarct size $\left[F_{(3,20)}=10.80\right.$, $n=24, P=0.0002]$ (Figure 3B). At day 14 after MCAO, the PSE conditioning and the PE conditioning significantly reduced the infarct volume compared to that of the ischemia/reperfusion + standard condition group $(P<0.001$ vs. $P S E, P=0.042$ vs. $\mathrm{PE})$, while the ischemia/reperfusion $(\mathrm{I} / \mathrm{R})+\mathrm{SE}$ group exhibited trends of reducing the infarct size, but the effect was not significant $(P=0.124)$ (Figure 3B). These results demonstrated that the neuroprotective effect of reducing infarct volume after MCAO can not be achieved by SE alone.

\section{Enriched Conditioning Increased Microvessel Density}

To evaluate how different housing conditions affected poststroke angiogenesis, immunofluorescent staining of CD31, which is expressed in all cells within the vascular compartment and plays diverse roles in angiogenesis, platelet function, and thrombosis (Baumann et al., 2004), was employed. In all ischemia/reperfusion groups, many blood vessels were intensely stained by the CD31 monoclonal antibody around the ischemic region (Figure 4A). Quantitative analysis showed that the MVD of the rats was significantly affected by group $\left[F_{(4,25)}=79.70, n=30, P<0.001\right]$. The enriched housing conditions significantly increased the MVD of the rats compared to that of the rats in the standard housing group. The PSE condition produced a better result than the PE or SE condition alone $(P<0.001)$, while the PE condition showed substantially better results than the SE condition $(P=0.002$, Figure $\mathbf{4 B})$.

\section{Enriched Conditions Upregulated the Expression of Angiogenic Factors}

To identify the signaling pathways responsible for the enhanced angiogenesis, the expression of the growth factors VEGF and Ang-1/Tie-2, which are two types of vascular regulatory molecules crucial for vessel formation and maturation (Augustin et al., 2009), was analyzed by immunohistochemistry (Figures 5A,B), and q-PCR (Figure 5C) in the penumbra at 14 days after stroke. The immunochemical results showed that the levels of Ang-1 and Tie-2 were significantly higher in the ischemia/reperfusion groups than in the sham + standard condition group. The PE, SE, and PSE conditions increased Ang-1/Tie-2 expression compared to that in the ischemia/reperfusion + standard condition group (Figures 5A,B). The rats in the ischemia/reperfusion + PSE group had significantly higher Ang-1/Tie-2 levels than those in the PE and SE groups. Moreover, the PE condition also had a larger effect on upregulating Ang-1/Tie-2 expression than the SE condition.

The VEGF and Ang-1 mRNA expression levels were further examined by qRT-PCR at 14 days after MCAO. As shown in Figure 5C, the VEGF and Ang-1 mRNA levels showed the same trends among the five groups, with statistical results of $\left[F_{(4,25)}=115.78, n=30, P<0.001\right]$ and $\left[F_{(4,25)}=66.91, n=30, P<0.001\right]$, respectively. The rats in the 

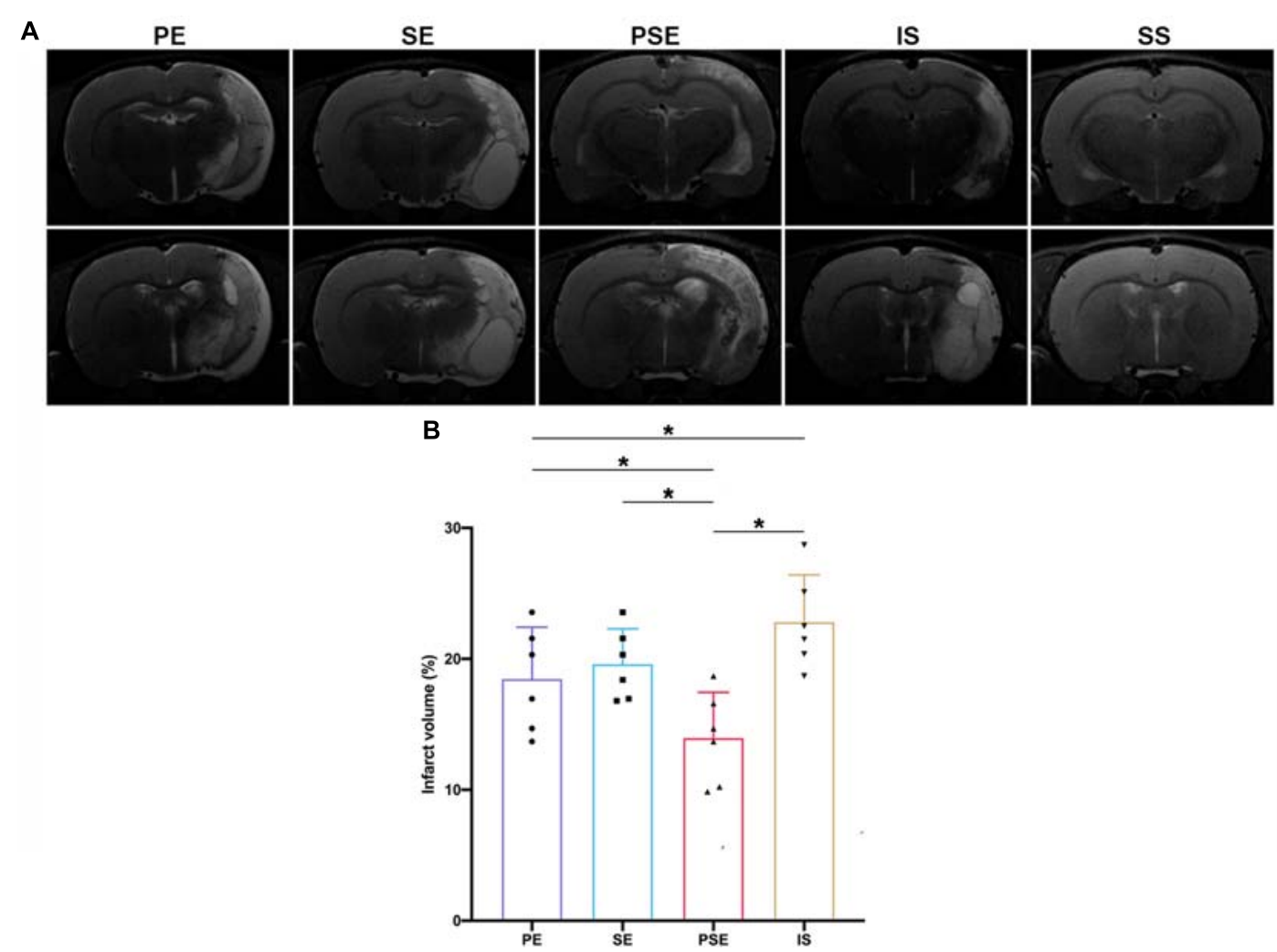

FIGURE 3 | Infarct volumes. (A) Representative images of different groups in T2 MRI scans at 14 days after stroke. Bregma levels were $-2.8 \mathrm{~mm}$ (the upper row) and $-5.2 \mathrm{~mm}$ (the lower row). (B) Quantitative analysis of infarct volumes according to T2 scans in four groups. $n=6$. Data are expressed as mean \pm SEM; ${ }^{*} P<0.05$.

ischemia/reperfusion + PE group, the ischemia/reperfusion $+\mathrm{SE}$ group and the ischemia/reperfusion + PSE group exhibited higher VEGF and Ang-1 expression than the rats in the ischemia/reperfusion + standard condition group $(P<0.01)$. The rats in the ischemia/reperfusion + PSE group had significantly higher VEGF/Ang-1 mRNA levels than those in the PE and SE groups, while the rats in the PE group had higher Ang-1 mRNA levels than those in the SE group $(P<0.001)$.

\section{DISCUSSION}

The model used was that of MCAO and Reperfusion. If the reperfusion was not performed, not many of the experimental animals would have survived till 14 days. Given the widely recognized effectiveness and complicated paradigms of $\mathrm{EE}$ treatment, the current study constructed different housing conditions for $\mathrm{I} / \mathrm{R}$ rats with the aim of determining whether a single or specific component of $\mathrm{EE}$ is sufficient to confer its neuroprotective benefits or if all components are necessary. The data suggest that the PE, SE, and PSE conditions demonstrated more advantages in promoting functional recovery and angiogenesis than the condition with no enrichment, but only exposure to the PSE condition yielded optimal benefits. This result is in accordance with previous research using a rat model of traumatic brain injury carried out by Sozda et al. (2010). However, the PE condition in the present study exhibited a larger effect on promoting neurological recovery than the SE condition, which is different from the results of the former study (Johansson and Ohlsson, 1996). This contradiction may be due to the different settings in these two studies: in the study by Johansson and Ohlsson, the rats in the $\mathrm{PE}$ group were housed individually with a running wheel, while the PE setting in the present study included much more stimulation.

\section{Intervention Duration}

In this study, the intervention time in the PE, SE, and PSE conditions was $4 \mathrm{~h}$ instead of $24 \mathrm{~h}$, which was out of consideration of the limitations of clinical treatment. It was reported that 6-h EE produced motor and cognitive benefits on traumatic brain injury similar to those of continuous EE (de Witt et al., 2011). Gaulke et al. (2005) reported that $1 \mathrm{~h}$ of EE per day was sufficient to augment cognitive recovery after fluid percussion brain injury. The study by Nygren and Wieloch (2005) showed that short $(3 \mathrm{~h})$ daily exposure was preferable to continued housing in EE because there was equal recovery of function and lower mortality. The disparity in the amount of EE necessary to confer functional recovery may be due to the different models of injury along with the diverse training protocols. In this study, 4-h exposure to enriched conditions exhibited better motor results after MCAO than the standard condition. The 


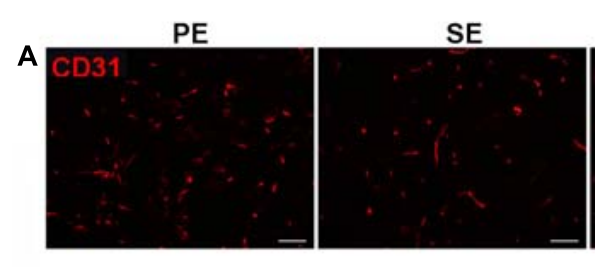

B

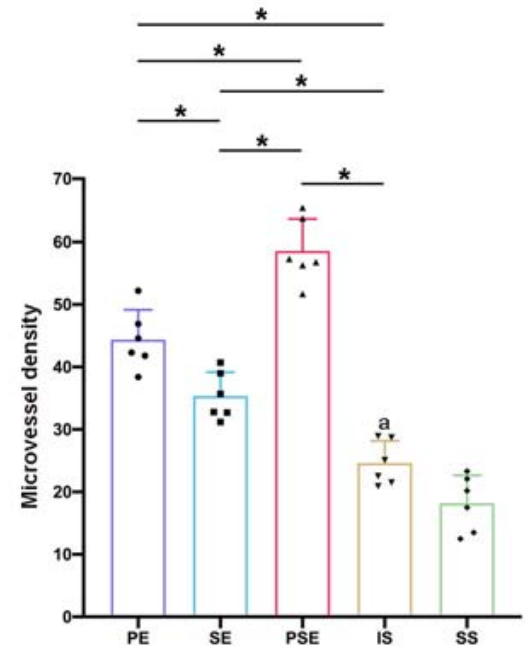

PSE
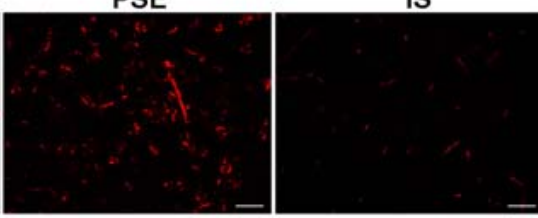

C ss
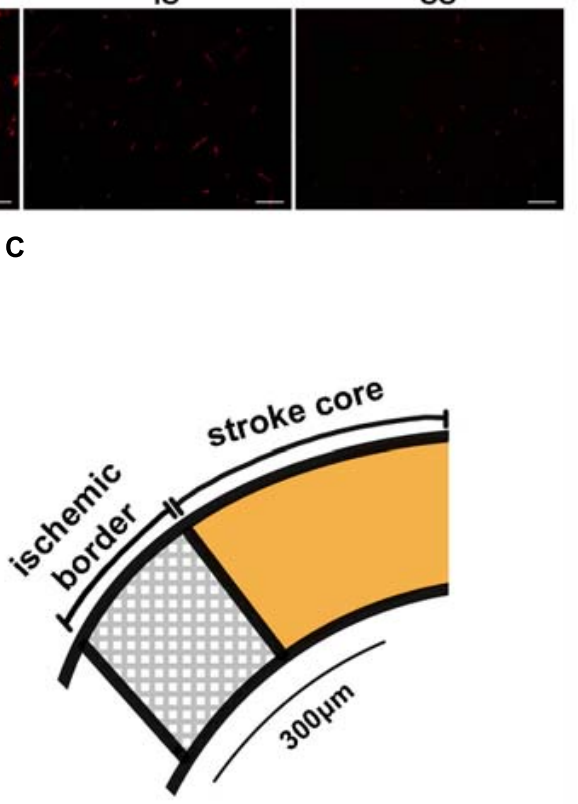

FIGURE 4 | Microvessel density (MVD) in the peri-infarct cortex at 14 days after MCAO. (A) Representative images of immunofluorescent labeling of CD31 in the periinfarct area. Bar $=50 \mu \mathrm{m}$. (B) Quantitative analysis of microvessel density (MVD). $n=6$. Data are expressed as mean \pm SEM; ${ }^{\star} P<0.05$. (C) Schematic brain with a highlight of ischemic border.

findings may have clinical relevance: in an inpatient setting, the cognitive and physical stimulation provided by multidisciplinary therapy could be viewed as an EE for stroke patients. Social interaction with other patients as well as with nurses and other staff would lead to more stimulation in that environment than what the patient may receive in therapy alone. Considering the physical strength and accompanying stress or cognitive issues, it is quite important to find the optimal amount of exposure to rehabilitation in clinical and experimental settings that results in improved outcomes without the deleterious side effects of excessive treatment. Our study demonstrated that 4-h enrichment was capable of producing behavioral benefit compared to the standard condition. However, further study with multiple intervention times is needed to pursue the optimal duration of EE.

\section{Angiogenesis After Stroke}

During the long period of repair and restoration following ischemia/reperfusion injury, it is non-negligible that enhanced angiogenesis plays an important role in improving the neurological outcome. In $\mathrm{I} / \mathrm{R}$ animal models, endothelial cell proliferation occurs 12-24 h after MCAO (Hayashi et al., 2003). Capillary sprouting and new vessel growth have been reported to continue for at least 3 weeks in the penumbra (Zhang et al., 2002; Hayashi et al., 2003). In ischemic stroke patients, Krupinski et al. (1994) reported that angiogenic activity occurred at 3-4 days after stroke. They also noticed that high levels of new vessel formation following stroke were correlated with better functional recovery and prolonged survival. During the process of angiogenesis, new capillaries are formed through direct proliferation and migration of endothelial progenitor cells from pre-existing blood vessels (Wang et al., 2012). Previous findings revealed that the area of CD31-immunopositive cells was significantly increased around the infarct after 28 days of treadmill training (Matsuda et al., 2011). It was also reported that physical exercise might improve motor performance and obviously increase the number of neogenetic microvessels around the penumbra following ischemic stroke, which might be related to increased CD31 expression ( $\mathrm{Hu}$ et al., 2010; Yang et al., 2012). In the present study, we found that the PE, SE and PSE groups exhibited significantly increased MVD compared with that of the IS group following 14 days of intervention. The PSE condition increased MVD to a greater extent than the PE and SE conditions.

To further identify the possible mechanism underlying poststroke angiogenesis, the expression levels of some angiogenic factors were examined. VEGF plays important roles in promoting the formation of primitive tubular structures as well as neuroprotection following ischemic injury (Hermann and Zechariah, 2009; Ma et al., 2012). Whereas VEGF works at the acute stage after focal cerebral ischemia, Ang-1/Tie-2 exert their functions at later stages of vascular development by mediating the interactions of endothelial cells and pericytes 


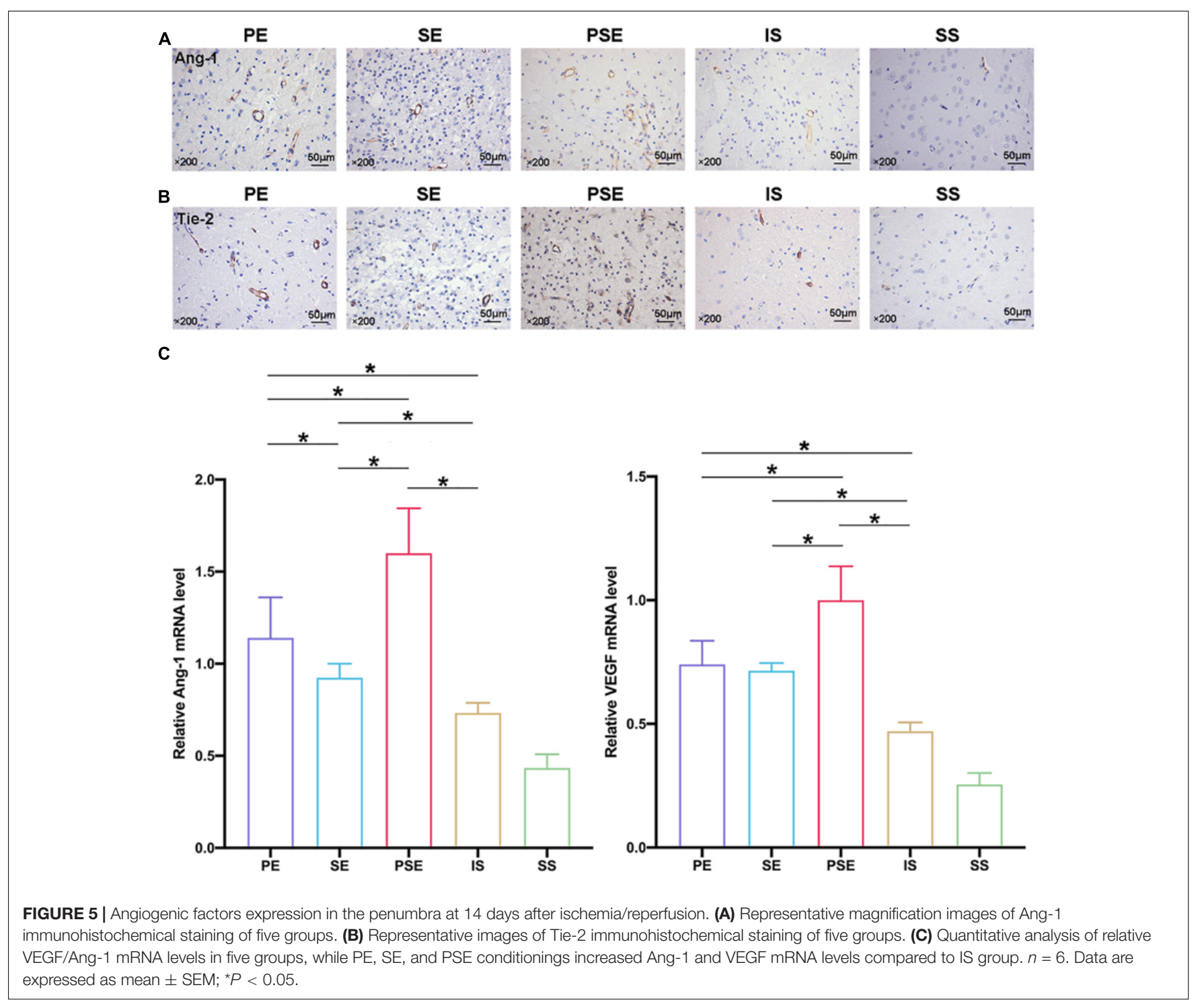

(Thurston et al., 1999) and promoting the maturation and stabilization of new vessels due to the formation of endothelial tight junctions and the recruitment of pericytes (Yancopoulos et al., 2000). Many reports have suggested that physical exercise and $\mathrm{EE}$ induced cerebral changes in the expression of angiogenic factors, such as VEGF/Ang-1 expression, which likely promote endothelial cell survival, proliferation, migration and tube formation (Seo et al., 2013; Gao et al., 2014). Social interaction could improve quality of life and decreases mortality after stroke (Boden-Albala et al., 2005). SE could decrease infarct size and brain atrophy, and increase neurogenesis and longterm functional recovery after ischemic stroke by facilitating social interaction (Fowler et al., 2002; Venna et al., 2014; Meng et al., 2015). However, the mechanisms underlying the promoting effects of SE on stroke rehabilitation is poorly understood. Our research showed that SE improved functional recovery and angiogenesis through the elevation of the expression of VEGF and Ang1/Tie 2. In the present study, we observed that cerebral ischemia-reperfusion induced angiogenic factor expression in the penumbra of MCAO rats, which showed that the PE, SE, and PSE conditions significantly upregulated angiogenic factor expression. The levation of these angiogenic factors could be due to a sequential process of increased metabolic demand associated with environments with different conditions. In addition, these findings are in line with the MVD observed in this study. Therefore, one possible mechanism for the EE-mediated enhancement of angiogenesis after stroke may be related to the upregulation of VEGF/Ang-1 expression. Some studies have shown that exposure to EE promoted angiogenesis after MCAO may be mediated by blood-borne factors such as hepatocyte growth factor (Xie et al., 2019). Other studies suggested that EE enhanced post-stroke angiogenesis via astrocytic HMGB1/IL-6 signaling pathway (Chen et al., 2017). There were also evidences that EE induced angiogenesis via regulating $\mathrm{PI} 3 \mathrm{~K} / \mathrm{AKT} / \mathrm{GSK}-3 / \beta$-catenin signaling pathway and activation of the intrinsic axonal guidance molecules in animal 
models of ischemic stroke (Zhan et al., 2020). However, the mechanism by which EE enhances angiogenesis after stroke needs further research.

The EEal conditions conclusively demonstrated more advantages in promoting functional recovery than no enrichment, but only exposure to the PSE condition, which contains all the components of a typical EE, yields the optimal benefits. Promoting angiogenesis may be one of the mechanisms underlying the neuroprotective effects of EEs. The findings of this study may have important translational relevance for clinical rehabilitation.

\section{DATA AVAILABILITY STATEMENT}

The original contributions presented in the study are included in the article/supplementary material, further inquiries can be directed to the corresponding author/s.

\section{ETHICS STATEMENT}

The animal study was reviewed and approved by the Institutional Animal Care and Use Committee of Wuhan University (approval number: WP2020-08059).

\section{REFERENCES}

Augustin, H. G., Koh, G. Y., Thurston, G., and Alitalo, K. (2009). Control of vascular morphogenesis and homeostasis through the angiopoietin-tie system. Nat. Rev. Mol. Cell Biol. 10, 165-177. doi: 10.1038/nrm2639

Baumann, C. I., Bailey, A. S., Li, W., Ferkowicz, M. J., Yoder, M. C., and Fleming, W. H. (2004). PECAM-1 is expressed on hematopoietic stem cells throughout ontogeny and identifies a population of erythroid progenitors. Blood 104, 1010-1016. doi: 10.1182/blood-2004-03-0989

Boden-Albala, B., Litwak, E., Elkind, M. S. V., Rundek, T., and Sacco, R. L. (2005). Social isolation and outcomes post stroke. Neurology 64, 1888-1892. doi: 10.1212/01.WNL.0000163510.79351.AF

Cassarino, M., and Setti, A. (2015). Environment as "brain training": a review of geographical and physical environmental influences on cognitive ageing. Ageing Res. Rev. 23, 167-182. doi: 10.1016/j.arr.2015.06.003

Chen, J.-Y., Yu, Y., Yuan, Y., Zhang, Y.-J., Fan, X.-P., Yuan, S.-Y., et al. (2017). Enriched housing promotes post-stroke functional recovery through astrocytic HMGB1-IL-6-mediated angiogenesis. Cell. Death Discov. 3:17054. doi: 10.1038/ cddiscovery.2017.54

Cirulli, F., Berry, A., Bonsignore, L. T., Capone, F., D’Andrea, I., Aloe, L., et al. (2010). Early life influences on emotional reactivity: evidence that social enrichment has greater effects than handling on anxiety-like behaviors, neuroendocrine responses to stress and central BDNF levels. Neurosci. Biobehav. Rev. 34, 808-820. doi: 10.1016/j.neubiorev.2010.02.008

Cui, X., Chen, J., Zacharek, A., Roberts, C., Yang, Y., and Chopp, M. (2009). Nitric oxide donor up-regulation of SDF1/CXCR4 and Ang1/Tie2 promotes neuroblast cell migration after stroke. J. Neurosci. Res. 87, 86-95. doi: 10.1002/ jnr.21836

de Witt, B. W., Ehrenberg, K. M., McAloon, R. L., Panos, A. H., Shaw, K. E., Raghavan, P. V., et al. (2011). Abbreviated environmental enrichment enhances neurobehavioral recovery comparably to continuous exposure after traumatic brain injury. Neurorehabil. Neural Repair 25, 343-350. doi: 10.1177/ 1545968310390520

\section{AUTHOR CONTRIBUTIONS}

$\mathrm{XZ}$ designed the study plan and carried out the tissue sampling, processing, and image acquisition, and drafted and edited the manuscript. JY-L provided technical support and participated in the discussion of the results. WJ-L and XP-C evaluated and revised the study design, supervised the execution of the research, and revised the manuscript. All authors contributed to the article and approved the submitted version.

\section{FUNDING}

This work was financially supported by the National Natural Science Foundation of China (no. 81902304), Zhongnan Hospital of Wuhan University Science, Technology and Innovation Seed Fund, Project: znpy2019113, and the Fundamental Research Funds for the Central Universities.

\section{ACKNOWLEDGMENTS}

We would like to thank the technical assistance given by National Center for Magnetic Resonance in Wuhan, State Key Laboratory of Magnetic Resonance and Atomic and Molecular Physics, Wuhan Institute of Physics and Mathematics, Chinese Academy of Sciences.

Diederich, K., Schmidt, A., Strecker, J.-K., Schäbitz, W.-R., Schilling, M., and Minnerup, J. (2014). Cortical photothrombotic infarcts impair the recall of previously acquired memories but spare the formation of new ones. Stroke 45, 614-618. doi: 10.1161/STROKEAHA.113.001907

Doeppner, T. R., Kaltwasser, B., Bähr, M., and Hermann, D. M. (2014). Effects of neural progenitor cells on post-stroke neurological impairment-a detailed and comprehensive analysis of behavioral tests. Front. Cell. Neurosci. 8:338. doi: 10.3389/fncel.2014.00338

Fone, K. C. F., and Porkess, M. V. (2008). Behavioural and neurochemical effects of post-weaning social isolation in rodents-relevance to developmental neuropsychiatric disorders. Neurosci. Biobehav. Rev. 32, 1087-1102. doi: 10. 1016/j.neubiorev.2008.03.003

Font, M. A., Arboix, A., and Krupinski, J. (2010). Angiogenesis, neurogenesis and neuroplasticity in ischemic stroke. Curr. Cardiol. Rev. 6, 238-244. doi: 10.2174/157340310791658802

Fowler, C. D., Liu, Y., Ouimet, C., and Wang, Z. (2002). The effects of social environment on adult neurogenesis in the female prairie vole. J. Neurobiol. 51, 115-128. doi: 10.1002/neu.10042

Fuss, J., Ben Abdallah, N. M.-B., Vogt, M. A., Touma, C., Pacifici, P. G., Palme, R., et al. (2010). Voluntary exercise induces anxiety-like behavior in adult C57BL/6J mice correlating with hippocampal neurogenesis. Hippocampus 20, 364-376. doi: 10.1002/hipo.20634

Gao, Y., Zhao, Y., Pan, J., Yang, L., Huang, T., Feng, X., et al. (2014). Treadmill exercise promotes angiogenesis in the ischemic penumbra of rat brains through caveolin-1/VEGF signaling pathways. Brain Res. 1585, 83-90. doi: 10.1016/j. brainres.2014.08.032

Gaulke, L. J., Horner, P. J., Fink, A. J., McNamara, C. L., and Hicks, R. R. (2005). Environmental enrichment increases progenitor cell survival in the dentate gyrus following lateral fluid percussion injury. Brain Res. Mol. Brain Res. 141, 138-150. doi: 10.1016/j.molbrainres.2005.08.011

Gertz, K., Priller, J., Kronenberg, G., Fink, K. B., Winter, B., Schröck, H., et al. (2006). Physical activity improves long-term stroke outcome via endothelial nitric oxide synthase-dependent augmentation of neovascularization and 
cerebral blood flow. Circ. Res. 99, 1132-1140. doi: 10.1161/01.RES.0000250175. 14861.77

Grégoire, C.-A., Bonenfant, D., Le Nguyen, A., Aumont, A., and Fernandes, K. J. L. (2014). Untangling the influences of voluntary running, environmental complexity, social housing and stress on adult hippocampal neurogenesis. PloS One 9:e86237. doi: 10.1371/journal.pone.0086237

Hannan, A. J. (2014). Environmental enrichment and brain repair: harnessing the therapeutic effects of cognitive stimulation and physical activity to enhance experience-dependent plasticity. Neuropathol. Appl. Neurobiol. 40, 13-25. doi: 10.1111/nan.12102

Hayashi, T., Noshita, N., Sugawara, T., and Chan, P. H. (2003). Temporal profile of angiogenesis and expression of related genes in the brain after ischemia. J. Cereb. Blood Flow Metab. 23, 166-180. doi: 10.1097/01.WCB.0000041283. 53351.CB

Hermann, D. M., and Zechariah, A. (2009). Implications of vascular endothelial growth factor for postischemic neurovascular remodeling. J. Cereb. Blood Flow Metab. 29, 1620-1643. doi: 10.1038/jcbfm.2009.100

Hu, X., Zheng, H., Yan, T., Pan, S., Fang, J., Jiang, R., et al. (2010). Physical exercise induces expression of CD31 and facilitates neural function recovery in rats with focal cerebral infarction. Neurol. Res. 32, 397-402. doi: 10.1179/ 016164110X12670144526309

Jin, Q., Cheng, J., Liu, Y., Wu, J., Wang, X., Wei, S., et al. (2014). Improvement of functional recovery by chronic metformin treatment is associated with enhanced alternative activation of microglia/macrophages and increased angiogenesis and neurogenesis following experimental stroke. Brain. Behav. Immun. 40, 131-142. doi: 10.1016/j.bbi.2014.03.003

Johansson, B. B., and Ohlsson, A. L. (1996). Environment, social interaction, and physical activity as determinants of functional outcome after cerebral infarction in the rat. Exp. Neurol. 139, 322-327. doi: 10.1006/exnr.1996.0106

Koellhoffer, E. C., and McCullough, L. D. (2013). The effects of estrogen in ischemic stroke. Transl. Stroke Res. 4, 390-401. doi: 10.1007/s12975-0120230-5

Krupinski, J., Kaluza, J., Kumar, P., Kumar, S., and Wang, J. M. (1994). Role of angiogenesis in patients with cerebral ischemic stroke. Stroke 25, 1794-1798. doi: 10.1161/01.str.25.9.1794

Lacar, B., Herman, P., Platel, J.-C., Kubera, C., Hyder, F., and Bordey, A. (2012). Neural progenitor cells regulate capillary blood flow in the postnatal subventricular zone. J. Neurosci. 32, 16435-16448. doi: 10.1523/JNEUROSCI. 1457- 12.2012

Leasure, J. L., and Decker, L. (2009). Social isolation prevents exercise-induced proliferation of hippocampal progenitor cells in female rats. Hippocampus 19, 907-912. doi: 10.1002/hipo.20563

Longa, E. Z., Weinstein, P. R., Carlson, S., and Cummins, R. (1989). Reversible middle cerebral artery occlusion without craniectomy in rats. Stroke 20, 84-91. doi: 10.1161/01.str.20.1.84

Luo, C. X., Jiang, J., Zhou, Q. G., Zhu, X. J., Wang, W., Zhang, Z. J., et al. (2007). Voluntary exercise-induced neurogenesis in the postischemic dentate gyrus is associated with spatial memory recovery from stroke. J. Neurosci. Res. 85, 1637-1646. doi: 10.1002/jnr.21317

Ma, Y., Zechariah, A., Qu, Y., and Hermann, D. M. (2012). Effects of vascular endothelial growth factor in ischemic stroke. J. Neurosci. Res. 90, 1873-1882. doi: $10.1002 /$ jnr. 23088

Matsuda, F., Sakakima, H., and Yoshida, Y. (2011). The effects of early exercise on brain damage and recovery after focal cerebral infarction in rats. Acta Physiol. Oxf. Engl. 201, 275-287. doi: 10.1111/j.1748-1708.2010.02174.x

Meng, C., Zhang, J.-C., Shi, R.-L., Zhang, S.-H., and Yuan, S.-Y. (2015). Inhibition of interleukin- 6 abolishes the promoting effects of pair housing on post-stroke neurogenesis. Neuroscience 307, 160-170. doi: 10.1016/j.neuroscience.2015.08. 055

Nygren, J., and Wieloch, T. (2005). Enriched environment enhances recovery of motor function after focal ischemia in mice, and downregulates the transcription factor NGFI-A. J. Cereb. Blood Flow Metab. 25, 1625-1633. doi: 10.1038/sj.jcbfm.9600157

Pang, T. Y. C., and Hannan, A. J. (2013). Enhancement of cognitive function in models of brain disease through environmental enrichment and physical activity. Neuropharmacology 64, 515-528. doi: 10.1016/j.neuropharm.2012.06. 029
Petcu, E. B., Smith, R. A., Miroiu, R. I., and Opris, M. M. (2010). Angiogenesis in old-aged subjects after ischemic stroke: a cautionary note for investigators. J. Angiogenesis Res. 2:26. doi: 10.1186/2040-2384-2-26

Ruan, L., Wang, B., ZhuGe, Q., and Jin, K. (2015). Coupling of neurogenesis and angiogenesis after ischemic stroke. Brain Res. 1623, 166-173. doi: 10.1016/j. brainres.2015.02.042

Rust, R., Grönnert, L., Gantner, C., Enzler, A., Mulders, G., Weber, R. Z., et al. (2019). Nogo-A targeted therapy promotes vascular repair and functional recovery following stroke. Proc. Natl. Acad. Sci. U. S. A. 116, 14270-14279. doi: 10.1073/pnas.1905309116

Seo, J. H., Yu, J. H., Suh, H., Kim, M.-S., and Cho, S.-R. (2013). Fibroblast growth factor- 2 induced by enriched environment enhances angiogenesis and motor function in chronic hypoxic-ischemic brain injury. PloS One 8:e74405. doi: 10.1371/journal.pone.0074405

Silasi, G., Hamilton, D. A., and Kolb, B. (2008). Social instability blocks functional restitution following motor cortex stroke in rats. Behav. Brain Res. 188, 219-226. doi: 10.1016/j.bbr.2007.10.030

Sozda, C. N., Hoffman, A. N., Olsen, A. S., Cheng, J. P., Zafonte, R. D., and Kline, A. E. (2010). Empirical comparison of typical and atypical environmental enrichment paradigms on functional and histological outcome after experimental traumatic brain injury. J. Neurotrauma 27, 1047-1057. doi: 10.1089/neu.2010.1313

Thurston, G., Suri, C., Smith, K., McClain, J., Sato, T. N., Yancopoulos, G. D., et al. (1999). Leakage-resistant blood vessels in mice transgenically overexpressing angiopoietin-1. Science 286, 2511-2514. doi: 10.1126/science.286.5449. 2511

Venna, V. R., Xu, Y., Doran, S. J., Patrizz, A., and McCullough, L. D. (2014). Social interaction plays a critical role in neurogenesis and recovery after stroke. Transl. Psychiatry 4:e351. doi: 10.1038/tp.2013.128

Voss, M. W., Vivar, C., Kramer, A. F., and van Praag, H. (2013). Bridging animal and human models of exercise-induced brain plasticity. Trends Cogn. Sci. 17, 525-544. doi: 10.1016/j.tics.2013.08.001

Wadowska, M., Woods, J., Rogozinska, M., and Briones, T. L. (2015). Neuroprotective effects of enriched environment housing after transient global cerebral ischaemia are associated with the upregulation of insulin-like growth factor-1 signalling. Neuropathol. Appl. Neurobiol. 41, 544-556. doi: 10.1111/ nan. 12146

Walker, M. D., and Mason, G. (2011). Female C57BL/6 mice show consistent individual differences in spontaneous interaction with environmental enrichment that are predicted by neophobia. Behav. Brain Res. 224, 207-212. doi: 10.1016/j.bbr.2011.06.003

Wang, Z., Tsai, L.-K., Munasinghe, J., Leng, Y., Fessler, E. B., Chibane, F., et al. (2012). Chronic valproate treatment enhances postischemic angiogenesis and promotes functional recovery in a rat model of ischemic stroke. Stroke 43, 2430-2436. doi: 10.1161/STROKEAHA.112.652545

Will, B., Galani, R., Kelche, C., and Rosenzweig, M. R. (2004). Recovery from brain injury in animals: relative efficacy of environmental enrichment, physical exercise or formal training (1990-2002). Prog. Neurobiol. 72, 167-182. doi: 10.1016/j.pneurobio.2004.03.001

Xie, H., Wu, Y., Jia, J., Liu, G., Zhang, Q., Yu, K., et al. (2013). Enrichment-induced exercise to quantify the effect of different housing conditions: a tool to standardize enriched environment protocols. Behav. Brain Res. 249, 81-89. doi: 10.1016/j.bbr.2013. 04.032

Xie, H., Yu, K., Zhou, N., Shen, X., Tian, S., Zhang, B., et al. (2019). Enriched environment elicits proangiogenic mechanisms after focal cerebral ischemia. Transl. Stroke Res. 10, 150-159. doi: 10.1007/s12975-018-0 629-8

Yancopoulos, G. D., Davis, S., Gale, N. W., Rudge, J. S., Wiegand, S. J., and Holash, J. (2000). Vascular-specific growth factors and blood vessel formation. Nature 407, 242-248. doi: 10.1038/35025215

Yang, Y.-R., Chang, H.-C., Wang, P. S., and Wang, R.-Y. (2012). Motor performance improved by exercises in cerebral ischemic rats. J. Mot. Behav. 44, 97-103. doi: 10.1080/00222895.2012.654524

Yu, K., Wu, Y., Zhang, Q., Xie, H., Liu, G., Guo, Z., et al. (2014). Enriched environment induces angiogenesis and improves neural function outcomes in rat stroke model. J. Neurol. Sci. 347, 275-280. doi: 10.1016/j.jns.2014.10.022 
Zhan, Y., Li, M.-Z., Yang, L., Feng, X.-F., Lei, J.-F., Zhang, N., et al. (2020). The three-phase enriched environment paradigm promotes neurovascular restorative and prevents learning impairment after ischemic stroke in rats. Neurobiol. Dis. 146:105091. doi: 10.1016/j.nbd.2020.105091

Zhang, F., Jia, J., Wu, Y., Hu, Y., and Wang, Y. (2010). The effect of treadmill training pre-exercise on glutamate receptor expression in rats after cerebral ischemia. Int. J. Mol. Sci. 11, 2658-2669. doi: 10.3390/ijms11072658

Zhang, P., Yu, H., Zhou, N., Zhang, J., Wu, Y., Zhang, Y., et al. (2013). Early exercise improves cerebral blood flow through increased angiogenesis in experimental stroke rat model. J. Neuroeng. Rehabil. 10:43. doi: 10.1186/1743-0003$10-43$

Zhang, X., Chen, X.-P., Lin, J.-B., Xiong, Y., Liao, W.-J., and Wan, Q. (2017). Effect of enriched environment on angiogenesis and neurological functions in rats with focal cerebral ischemia. Brain Res. 1655, 176-185. doi: 10.1016/j.brainres. 2016.11.001
Zhang, Z. G., Zhang, L., Croll, S. D., and Chopp, M. (2002). Angiopoietin-1 reduces cerebral blood vessel leakage and ischemic lesion volume after focal cerebral embolic ischemia in mice. Neuroscience 113, 683-687. doi: 10.1016/ s0306-4522(02)00175-6

Conflict of Interest: The authors declare that the research was conducted in the absence of any commercial or financial relationships that could be construed as a potential conflict of interest.

Copyright (c) 2021 Zhang, Liu, Liao and Chen. This is an open-access article distributed under the terms of the Creative Commons Attribution License (CC BY).

The use, distribution or reproduction in other forums is permitted, provided the original author(s) and the copyright owner(s) are credited and that the original publication in this journal is cited, in accordance with accepted academic practice. No use, distribution or reproduction is permitted which does not comply with these terms. 